\title{
MEET-IRREDUCIBLE ELEMENTS IN IMPLICATIVE LATTICES ${ }^{1}$
}

\author{
DOROTHY P. SMITH
}

\begin{abstract}
A characterization of meet-irreducible elements and atoms in an implicative lattice is obtained and used to derive the following theorems. A complete lattice is implicative and every element has a meet-irreducible decomposition if and only if there are enough principal prime relative annihilator ideals to separate distinct elements. The MacNeille completion of an implicative lattice is an implicative lattice; furthermore the embedding preserves relative pseudocomplements, meet-irreducible elements and atoms.
\end{abstract}

1. Introduction. An implicative lattice is a system $\mathfrak{L}=(L, \leqq, \vee, \wedge, \rightarrow, 1)$ such that $(L, \vee, \wedge)$ is a lattice and $\rightarrow$ is a binary operation on $L$ which, for every $a, b, c \in L$, satisfies

$$
c \leqq a \rightarrow b \text { if and only if } c \wedge a \leqq b .
$$

The element $a \rightarrow b$ is called the pseudocomplement of a relative to $b$. The variety of implicative lattices and its relationship to logic and other mathematical systems has been extensively studied [2], [8], [9], [11], [12] with considerable lack of uniformity in terminology and notation. The following is a brief list of properties for later reference.

If $\mathfrak{L}$ is an implicative lattice and $x, y, z \in L$, then

(1.1) $y \leqq x \rightarrow y$;

(1.2) $x \leqq y$ if and only if $x \rightarrow y=1$;

(1.3) $x \leqq y$ implies $z \rightarrow x \leqq z \rightarrow y$ and $y \rightarrow z \leqq x \rightarrow z$;

(1.4) $(y \wedge z) \rightarrow x=z \rightarrow(y \rightarrow x)=y \rightarrow(z \rightarrow x)=(z \rightarrow y) \rightarrow(z \rightarrow x)$;

(1.5) $L$ is a distributive lattice;

(1.6) if $B \subseteq L$ such that $\bigwedge B$ exists then, for every $a \in L, \bigwedge\{a \rightarrow y \mid y \in B\}$ exists and equals $a \rightarrow \wedge B[11$, p. 136].

2. Meet-irreducible elements and atoms. In 1938, Ward [13] obtained a necessary condition for an element to be meet-irreducible in a residuated

Presented to the Society, January 24, 1969 under the title Some results on Brouwerian lattices; received by the editors August 4, 1971.

AMS 1970 subject classifications. Primary 06A25; Secondary 06A35.

Key words and phrases. Brouwerian lattice, Heyting algebra, implicative lattice, lattice, meet-irreducible, relative annihilator, relative pseudocomplement.

1 These results are contained in the author's doctoral dissertation written with the guidance of Professor Don E. Edmondson at the University of Texas at Austin.

(c) American Mathematical Society 1972 
lattice. The following theorem shows this condition to be both necessary and sufficient for an element to be meet-irreducible in an implicative lattice.

THEOREM 2.1. An element $m$ is meet-irreducible in an implicative lattice $\mathfrak{L}$ if and only if $x \mathrm{t}$ implies $x \rightarrow m=m$.

Proof of NECESSITY. Let $m$ be a meet-irreducible element of an implicative lattice $\mathfrak{L}$ and $x \in L$ such that $x \leq m$. By definition of $x \rightarrow m$ it will suffice to show that $x \wedge z \leqq m$ implies $z \leqq m$, since clearly $z \leqq m$ implies $x \wedge z \leqq m$. If $x \wedge z \leqq m$ then $m=m \vee(x \wedge z)=(m \vee x) \wedge(m \vee z)$, so $m=m \vee x$ or $m=m \vee z$ since $m$ is meet-irreducible, but $x \neq m$ implies $m \neq m \vee x$ thus $m=m \vee z$, i.e. $z \leqq m$.

Proof OF SUFFICIENCY. Let $m \in L$ such that, for every $x \leq m, x \rightarrow m=m$ and $p, q \in L$ such that $m<p$ and $m=p \wedge q$. Then $p \leq m$ so $p \rightarrow m=m=p \wedge q$, so by definition of $p \rightarrow m q \leqq m$, but also $m=p \wedge q \leqq q$, hence $m=q$; therefore $m$ is meet-irreducible.

COROllary 2.1.a. An element $m$ is meet-irreducible in a Boolean algebra if and only if $x \leq m$ implies $m^{\prime} \leqq x$.

COROLlaRY 2.1.b. If $b$ is meet-irreducible then, for every $a, a \rightarrow b$ is meet-irreducible.

Proof. If $x \leq a \rightarrow b$ then $x \leq b \leqq a \rightarrow b$ so $x \rightarrow b=b$; hence $x \rightarrow(a \rightarrow b)=$ $a \rightarrow(x \rightarrow b)=a \rightarrow b$, by (1.4).

COROLlaRY 2.1.c. If $\tau: L_{1} \rightarrow L_{2}$ is an implicative homomorphism and $m$ is a meet-irreducible element of $L_{1}$ then $m \tau$ is a meet-irreducible element of $L_{1} \tau$.

Proof. If $x \tau \in L_{1} \tau$ such that $x \tau+m \tau$ then $x \leq m$ hence $x \rightarrow m=m$ and $(x \rightarrow m) \tau=x \tau \rightarrow m \tau=m \tau$.

COROLlaRY 2.1.d. If $M$ is a set of meet-irreducible elements and $b=$ $\bigwedge M$ then, for every $a, a \rightarrow b=\bigwedge\{a \rightarrow m \mid m \in M\}=\bigwedge\{m \mid m \in M$ and $a \leq m\}$.

Let $\mathfrak{L}=(L, \vee, \wedge, \rightarrow, 1,0)$ be a pseudo-Boolean algebra, i.e. an implicative lattice with least element, 0 , and $x^{*}=x \rightarrow 0$ denote the pseudocomplement of $x$.

THEOREM 2.2. An element $a$ is an atom in a pseudo-Boolean algebra if and only if $a \neq 0$ and $a \neq y$ implies $a \rightarrow y=a^{*}$.

ProOF OF NECESSITY. Let $a$ be an atom and $y \in L$ such that $a \leq y$. It will suffice to show that $z \leqq a^{*}$ if and only if $z \wedge a \leqq y$. If $z \leqq a^{*}$ then $z \wedge a=0$; so, for every $y, z \wedge a \leqq y$. Conversely, if $z \wedge a \leqq y$ then since $a$ is an atom $z \wedge a=0$ or $z \wedge a=a$, but $a=z \wedge a \leqq y$ contradicts $a \leq y$; thus $0=z \wedge a$, i.e. $z \leqq a^{*}$. 
Proof OF SUfFICIENCY. Let $a \neq 0$, for every $y, a \neq y$ implies $a \rightarrow y=a^{*}$, and $z<a$. Then $z=z \wedge a$ and $a \leq z$ so $z \leqq a \rightarrow z=a^{*}$; hence $z=z \wedge a=0$, i.e. $a$ is an atom.

Corollary 2.2.a. If $a$ is an atom then $a^{*}$ is meet-irreducible.

Proof. First note that $x \leq a^{*}$ if and only if $a x^{*}$; so if $a$ is an atom and $x \neq a^{*}$ then $a \rightarrow x^{*}=a^{*}$, so by (1.4), $x \rightarrow a^{*}=x \rightarrow\left(a \rightarrow x^{*}\right)=a \rightarrow\left(x \rightarrow x^{*}\right)=$ $a \rightarrow x^{*}=a^{*}$; thus $a^{*}$ is meet-irreducible by Theorem 2.1 .

3. Meet-irreducible decompositions and relative annihilator ideals. Balachandran [1, p. 550] has shown that a complete lattice is implicative if every element has a finite meet-prime decomposition and Newman [10, p. 31] that a complete distributive lattice is meet-continuous if every element has a finite meet-irreducible decomposition. The next theorem generalizes these results.

THEOREM 3.1. If every element of a complete distributive lattice has a meet-irreducible decomposition, then the lattice is implicative.

Proof. Let $\mathfrak{L}$ be a complete distributive lattice in which every element has a meet-irreducible decomposition, $M$ be the set of meet-irreducible elements and, for each $x \in L, M(x)=\{m \in M \mid x \leqq m\}$. Since $\mathfrak{L}$ is complete, a well-defined binary operation is obtained by defining, for every $a, b \in L$, $a \rightarrow b=\wedge[M(b)-M(a)]$. Observe that, for every $x \in L, 1 \in M(x)$, so $a \rightarrow b=1$ if and only if $M(b)-M(a)=\varnothing$ if and only if $a \leqq b$.

McKinsey and Tarski [8, Theorem 1.4] have shown that $\mathfrak{E}$ is implicative provided, for every $a, b, c \in L$, (1) $a \wedge a \rightarrow b \leqq b$, (2) $a \leqq b \rightarrow(a \wedge b)$, and (3) $c \rightarrow(a \wedge b) \leqq c \rightarrow a$. First note that $a \wedge a \rightarrow b \leqq b$ is implied by $M(b) \subseteq M(a) \cup$ $[M(b)-M(a)]$, so (1) is established. To establish (2), we use the distributivity to show that $M(a \wedge b)=M(a) \cup M(b)$; for if $q \in M(a \wedge b)$ then $q=(a \wedge b) \vee q=$ $(a \vee q) \wedge(b \vee q)$, so $q=a \vee q$ or $q=b \vee q$, i.e. $q \in M(a) \cup M(b)$; from this it follows that $M(a \wedge b)-M(b) \subseteq M(a)$, so $a=\wedge M(a) \leqq \wedge[M(a \wedge b)-M(b)]$ $=b \rightarrow(a \wedge b)$. Finally, $M(a) \subseteq M(a \wedge b)$, so $M(a)-M(c) \subseteq M(a \wedge b)-M(c)$ and $c \rightarrow(a \wedge b)=\bigwedge[M(c)-M(a \wedge b)] \leqq \wedge[M(a)-M(c)]=c \rightarrow a$.

Mandelker [7] introduced the notion of the annihilator of a relative to $b=\langle a, b\rangle=\{z \mid z \wedge a \leqq b\}$ and showed that a lattice is distributive if and only if every relative annihilator is an ideal. Clearly, a lattice is implicative if and only if every relative annihilator is a principal ideal, in which case $\langle a, b\rangle=(a \rightarrow b]$. Note further that in a distributive lattice there are enough relative annihilator ideals to separate distinct elements, since $a+b$ implies $b \in\langle a, b\rangle$ but $a \notin\langle a, b\rangle$. Also the relative annihilator $\langle a, b\rangle$ is proper if and only if $a \leq b$, so the class of relative annihilators needed to separate elements need contain none for which $a \leqq b$. 
THEOREM 3.2. Let $\mathfrak{L}$ be a complete lattice. Then $\mathfrak{L}$ is an implicative lattice in which every element has a meet-irreducible decomposition if and only if there are enough principal prime relative annihilator ideals to separate distinct elements.

Proof. If there are enough principal prime relative annihilator ideals to separate elements then there are enough prime ideals to separate elements; hence $L$ is distributive and the principal ideal ( $p]$ is prime if and only if $p$ is meet-irreducible. For each $x \in L$, let $M(x)=\{m \in L \mid m$ is meetirreducible and $x \leqq m\}$, so $\bigwedge M(x)=\bar{x}$ exists in $L$ and $x \leqq \bar{x}$; but if $x<\bar{x}$ then there exists a principal prime ideal ( $p$ ] such that $x \in(p]$ but $\bar{x} \notin(p]$; hence there exists a meet-irreducible element $p$ such that $p \in M(x)$ but $\bar{x} \neq p$ contradicting $\bar{x}=M(x)$; hence $x=\wedge M(x)$, and, by Theorem $3.1, \mathfrak{L}$ is implicative.

Conversely, suppose $L$ is an implicative lattice in which every element has a meet-irreducible decomposition and $b=\wedge M(b) \leq a=\wedge M(a)$. Then, for some $\bar{m} \in M(a), \bar{m} \notin M(b)$, so $b \leq \bar{m}$ and $a \leqq \bar{m} \leqq b \rightarrow \bar{m}$; thus $a \in\langle b, \bar{m}\rangle=(\bar{m}]$ and $b \notin\langle b, \bar{m}\rangle=(\bar{m}]$.

4. The MacNeille completion. MacNeille [6] showed that by generalizing Dedekind's completion by cuts of the rational numbers, any partially ordered set can be embedded in a complete lattice in a manner which preserves the order relation and any existing greatest lower bounds and least upper bounds (i.e. it is a regular embedding). Furthermore, the embedding is both meet and join dense. The embedding does not necessarily preserve other important lattice structure; Funayama [4] constructed a distributive lattice whose MacNeille completion was not even modular, and Crawley [3] constructed a distributive lattice which could not be regularly embedded in any complete modular lattice. The existence of complements or relative pseudocomplements seems however to be helpful in preserving distributivity, as Glivenko [5] and Stone [12] show that the MacNeille completion of a Boolean algebra is a Boolean algebra and we now show that the MacNeille completion of an implicative lattice is an implicative lattice; furthermore the embedding preserves relative pseudocomplements and any existing meet-irreducible elements and atoms.

THEOREM 4.1. The MacNeille completion of an implicative lattice is an implicative lattice, and the embedding preserves relative pseudocomplements.

Proof. Let $\mathfrak{L}$ be an implicative lattice and $\mathfrak{L}_{e}$ denote its MacNeille completion. Recall [2, Chapter V, §9] that $L_{e}=\left\{A^{+} \mid A \subseteq L\right\}$ where $A^{+}$is the set of lower bounds of $A$ in $\mathfrak{L}$, set inclusion is the partial order and set intersection is the meet operation, and the embedding is $x \rightarrow(x]$, the 
principal ideal generated by $x$. It is easily shown that, for every $B, C \in L_{e}$,

$$
\begin{aligned}
B \wedge C & =\wedge\{(b \wedge c] \mid B \subseteq(b] \text { and } C \subseteq(c]\} \\
& =\bigvee\{(x \wedge y] \mid(x] \subseteq B \text { and }(y] \subseteq C\}
\end{aligned}
$$

Since $\mathfrak{L}_{e}$ is a complete láttice, a well-defined binary operation is obtained as follows: for $M, N \in L_{e}$,

$$
M \rightarrow N=\bigwedge\{(m \rightarrow n] \mid(m] \subseteq M \text { and } N \subseteq(n]\} .
$$

The binary operation satisfies the definition of a relative pseudocomplement, since $Z=\bigvee\{(z] \mid(z] \subseteq Z\} \subseteq M \rightarrow N$ iff, for every $(m] \subseteq M, N \subseteq(n]$ and $(z] \subseteq Z, z \leqq m \rightarrow n$ iff, for every $(m] \subseteq M, N \subseteq(n]$ and $(z] \subseteq Z, z \wedge m \leqq n$ iff

$Z \wedge M=\bigvee\{(z \wedge m] \mid(z] \subseteq Z$ and $(m] \subseteq M\} \subseteq \wedge\{(n] \mid N \subseteq(n]\}=N$.

Thus $\mathfrak{L}_{e}$ is an implicative lattice.

Furthermore, the embedding $x \rightarrow(x]$ is an implicative homomorphism, since, for every $a, b \in L,(a] \rightarrow(b]=\bigwedge\{(x \rightarrow y] \mid(x] \subseteq(a]$ and $(b] \subseteq(y]\}$; but, by (1.3), $x \leqq a$ implies $a \rightarrow b \leqq x \rightarrow b$ and $b \leqq y$ implies $x \rightarrow b \leqq x \rightarrow y$; hence $a \rightarrow b \leqq x \rightarrow y$; thus $(a] \rightarrow(b]=(a \rightarrow b]$.

Corollary 4.1.a. For every $b \in L$ and $X \in L_{e}$,

$$
X \rightarrow(b]=\bigwedge\{(x \rightarrow b] \mid x \in X\} \text { and }(b] \rightarrow X=\bigwedge\{(b \rightarrow y] \mid X \subseteq(y]\} .
$$

Proof. By the preceding theorem,

$$
X \rightarrow(b]=\bigwedge\{(x \rightarrow y] \mid(x] \subseteq X \text { and }(b] \subseteq(y]\},
$$

but $(b] \subseteq(y]$ if and only if $b \leqq y$ which implies that $x \rightarrow b \leqq x \rightarrow y$; also $X \in L_{e}$ if and only if $X=Y^{+}$for some $Y \subseteq L$ so $(x] \subseteq X$ if and only if $x \in X$; hence $X \rightarrow(b]=\bigwedge\{(x \rightarrow b] \mid x \in X\}$. Similarly, $(b] \rightarrow X=\bigwedge\{(z \rightarrow y] \mid(z] \subseteq(b]$ and $X \subseteq(y]\}=\bigwedge\{(b \rightarrow y] \mid X \subseteq(y]\}$ since $(z] \subseteq(b]$ if and only if $z \leqq b$ which implies $b \rightarrow y \leqq z \rightarrow y$.

COROLlaRY 4.1.b. If $m$ is a meet-irreducible element of $\mathfrak{L}$ then $(m]$ is a meet-irreducible element of $\mathfrak{L}_{e}$.

Proof. For any $X \in L_{e}$ such that $X \notin(m]$ there exists $x_{0} \in X$ such that $x_{0} \leq m$ so if $m$ is a meet-irreducible element of $L$ then, by Theorem 2.1, $x_{0} \rightarrow m=m$, and, by Corollary 4.1.a, $X \rightarrow(m]=\bigwedge\{(x \rightarrow m] \mid x \in X\}$ so $X \rightarrow(m) \subseteq\left(x_{0} \rightarrow m\right)=(m)$; but, by $(1.1),(m) \subseteq X \rightarrow(m) ;$ therefore $X \rightarrow(\dot{m})=$ $(m]$ and $(m]$ is a meet-irreducible element of $\mathfrak{L}_{e}$.

COROLlaRY 4.1.c. If every element of an implicative lattice has a meetirreducible decomposition then every element of its MacNeille completion has a meet-irreducible decomposition.

COROLLARY 4.1.d. If $a$ is an atom of $\mathfrak{L}$, then $(a]$ is an atom of $\mathfrak{L}_{e}$. 
Proof. For any $Y \in L_{e}$ such that $(a] \ddagger Y=Z^{+}$there exists $z_{0} \in Z$ such that $a \leq z_{0}$, so if $a$ is an atom of $\mathfrak{L}$, then, by Theorem 2.2, $a \rightarrow z_{0}=a^{*}$ and, by Corollary 4.1.a, $(a] \rightarrow Y \subseteq\left(a \rightarrow z_{0}\right)=\left(a^{*}\right]$; but $\left(a^{*}\right] \subseteq(a] \rightarrow Y$ since $\left(a^{*}\right] \wedge$ $(a]=(0] \subseteq Y$; therefore $(a] \rightarrow Y=\left(a^{*}\right]=(a]^{*}$ and $(a]$ is an atom of $\mathfrak{L}_{e}$.

COROLlary 4.1.e. The MacNeille completion of an atomic implicative lattice is atomic.

\section{REFERENCES}

1. V. K. Balachandran, On complete lattices and a problem of Birkhoff and Frink, Proc. Amer. Math. Soc. 9 (1955), 548-553. MR 17, 341.

2. G. Birkhoff, Lattice theory, 3rd ed., Amer. Math. Soc. Colloq. Publ., vol. 25, Amer. Math. Soc., Providence, R.I., 1967. MR 37 \#2638.

3. P. Crawley, Regular embeddings which preserve lattice structure, Proc. Amer. Math. Soc. 13 (1962), 748-752. MR 25 \#3871.

4. N. Funayama, On the completion by cuts of distributive lattices, Proc. Imp. Acad. Tokyo 20 (1944), 1-2. MR 7, 236.

5. V. Glivenko, Sur quelques points de la logique de Brouwer, Bull. Acad. Sci. Belgique 15 (1929), 183-188.

6. H. MacNeille, Partially ordered sets, Trans. Amer. Math. Soc. 42 (1937), 416-460.

7. M. Mandelker, Relative annihilators in lattices, Duke Math. J. 37 (1970), 377-386. MR 41 \#1606.

8. J. C. C. McKinsey and A. Tarski, On closed elements in closure algebras, Ann. of Math. (2) 47 (1946), 122-162. MR 7, 359.

9. W. C. Nemitz, Implicative semi-lattices, Trans. Amer. Math. Soc. 117 (1965), 128142. MR 31 \#1212.

10. T. Newman, Homomorphic images of compactly generated lattices, Doctoral Dissertation, University of Texas, Austin, Tex., 1967 (unpublished).

11. $\mathrm{H}$. Rasiowa and R. Sikorski, The mathematics of metamathematics, Monografie Mat., Tom 41, PWN, Warsaw, 1963. MR 29 \#1149.

12. M. H. Stone, Topological representation of distributive lattices and Brouwerian logics, Casopis Mat. Fys. 67 (1937), 1-25.

13. M. Ward, Structure residuation, Ann. of Math. (2) 39 (1938), 558-568.

Department of Mathematics, louisiana State University in New Orleans, New OrLeans, Louisiana 70122 